\title{
WHITNEY STABILITY AND CONTRACTIBLE HYPERSPACES
}

\author{
A. M. DILKS AND J. T. ROGERS, JR.
}

\begin{abstract}
A contraction technique of the first author is used to derive, for a certain class of continua with contractible hyperspaces, results about Whitney stability, the cone $=$ hyperspace property, and Whitney properties.
\end{abstract}

Let $C(X)$ be the hyperspace of subcontinua of the continuum $X$, and let $\mu$ be a Whitney map on $C(X)$. Kelley [6] has shown that if $C(X)$ is contractible, then the contraction can be chosen so that each subcontinuum of $X$ "grows" until it becomes $X$. The first author $[3,4]$ has improved this result by showing that the contraction may be chosen with the additional property that, at time $t$, the contraction has moved each point $A$ of $C(X)$ with $\mu(A) \leqslant t$ into the Whitney level $\mu^{-1}(t)$ and has left unmoved each point $A$ with $\mu(A)>t$.

In this paper, we exploit this contraction in several ways. We prove that if the continuum $X$ has property $\mathrm{K}$, has the covering property, and has arc components that are one-to-one continuous images of the real line such that both ends are dense in $X$, then the cone over $X$, Cone $(X)$, is homeomorphic to $C(X)$. Moreover, if $\mu$ is a Whitney map on $C(X)$, then the homeomorphism may be chosen so that its restriction to $X \times\{t\}$ is a homeomorphism onto $\mu^{-1}(t)$.

In particular, the base of the cone is mapped homeomorphically onto the singletons and the vertex onto the point $X$. Hence, such continua have the cone $=$ hyperspace property and are Whitney stable.

We give an example of a continuum with these properties that is neither arc-like nor circle-like. This gives a negative answer to Question 8.14 of [9]. The solenoids are examples of circle-like continua with these properties.

We turn our attention to the more general class of continua with property $\mathbf{K}$ and with the property that each proper, nondegenerate subcontinuum is an arc. We construct a monotone map of $\operatorname{Cone}(X)$ onto $C(X)$ similar to the homeomorphism above. In the case that $X$ is the inverse limit of arcs with open maps as the bonding maps, we approximate this monotone map with a level-preserving homeomorphism. It follows that such a continuum has the cone $=$ hyperspace property and is Whitney stable. This gives an affirmative answer to Question 14.22 of [9] and partial answers to Questions 8.11 and 8.15 of [9].

Modifications of these methods are used to show that the sin $1 / x$-curve and a ray spiralling on a circle do not admit essentially different Whitney maps.

Received by the editors December 12, 1980 and, in revised form, February 23, 1981.

1980 Mathematics Subject Classification. Primary 54B20.

Key words and phrases. Contractible hyperspace, Whitney stability, cone = hyperspace property, property $\mathbf{K}$, sliced decomposition. 
Throughout this paper, the letter $\mu$ will denote a Whitney map for the hyperspace $C(X)$. The symbol $\sigma$ will denote the map from $C(C(X))$ to $C(X)$ defined by $\sigma(\mathbb{Q})=\cup\{A: A \in \mathbb{Q}\}$, and $\sigma_{t}$ will denote the restriction of $\sigma$ to $C\left(\mu^{-1}(t)\right)$. The letter $G$ will denote the map of $X \times I$ to $C(X)$ defined as follows:

$$
G(a, t)=\sigma\left\{A \in \mu^{-1}(t): a \in A\right\} .
$$

Kelley [6] has shown that $G$ is continuous if the continuum $X$ has property $K$. Kelley [6] also defined $\mathcal{G}: C(X) \times I \rightarrow C(X)$ by

$$
\mathcal{G}(A, t)=\sigma\left\{G\left(a, t^{\prime}\right): a \in A, t^{\prime} \leqslant t\right\} .
$$

Then $\mathcal{G}$ restricted to the set of singletons is the map $G$, and $\mathcal{G}$ has the property that $\mathcal{G}\left(A, t_{1}\right) \subseteq \mathcal{G}\left(A, t_{2}\right)$ whenever $t_{1}<t_{2}$. Define $\tilde{\mathcal{G}}: C(X) \times I \rightarrow C(X)$ by

$$
\tilde{\mathcal{G}}(A, t)=\left\{\begin{array}{l}
A, \quad t \leqslant \mu(A), \\
\mathcal{G}(A,(t-\mu(A)) /(1-\mu(A))), \quad t>\mu(A), \mu(A)<1 .
\end{array}\right.
$$

Define $\tilde{H}: C(X) \times I \rightarrow C(X)$ by

$$
\tilde{H}(A, t)=\tilde{\mathcal{G}}\left(A, \inf \left\{s: \mathcal{G}(A, s) \in \mu^{-1}(t)\right\}\right) .
$$

Then $\tilde{\mathcal{G}}$ and $\tilde{H}$ are continuous [4 or 5]. Let $H: X \times I \rightarrow C(X)$ be the restriction of $\tilde{H}$ to the set of singletons in $C(X)$.

Denote $H$ restricted to $X \times\{t\}$ by $H_{t}$. Note that the range of $H_{t}$ is $\mu^{-1}(t)$.

Let $\hat{H}: \operatorname{Cone}(X) \rightarrow C(X)$ be the map induced by $H$. It is easily seen that $\hat{H}$ is continuous and that it is a homeomorphism provided each $H_{t}$ is a homeomorphism. If $\psi$ is the natural map from $X \times I$ to $\operatorname{Cone}(X)$, we have the following commutative diagram:

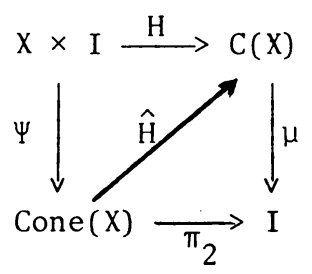

Diagram I

A continuum $X$ is said to be arc-like if it is the inverse limit of arcs with surjective bonding maps. The continuum $X$ is circle-like if it is the inverse limit of circles with surjective bounding maps.

A continuum $X$ is said to admit essentially different Whitney maps provided that there exist Whitney maps $\mu$ and $\mu^{\prime}$ for $C(X)$ such that some member of $\left\{\mu^{-1}(t)\right.$ : $0<t \leqslant \mu(X)\}$ or of $\left\{\left(\mu^{\prime}\right)^{-1}(t): 0<t<\mu^{\prime}(X)\right\}$ is not homeomorphic to any member of the other set.

A continuum is said to be Whitney stable provided that, for all Whitney maps $\mu$ for $C(X), \mu^{-1}(t)$ is homeomorphic to $X$ for each $t \in(0, \mu(X))$.

A continuum $X$ is said to have the covering property provided that no proper subcontinuum of $\mu^{-1}(t)$ covers $X$, for any Whitney map $\mu$ on $C(X)$ and any $t \in(0, \mu(X))$.

A map $f$ from $X$ onto $Y$ is said to be weakly confluent provided that, for each subcontinuum $K$ of $Y$, there exists a component $A$ of $f^{-1}(K)$ such that $f(A)=K$. 
A continuum $X$ is said to be in Class (W) provided that every mapping of any continuum onto $X$ is weakly confluent. Chainable continua are in Class (W). Being in Class (W) is equivalent to having the covering property.

A topological property $\mathbf{P}$ is said to be a Whitney property if whenever a continuum $X$ has property $\mathrm{P}$, then so does $\mu^{-1}(t)$ for each Whitney map $\mu$ on $C(X)$ and each $t \in(0, \mu(X))$.

A continuum $X$ is said to have the cone $=$ hyperspace property if there is a homeomorphism $h$ : Cone $(X) \rightarrow C(X)$ such that $h$ maps the vertex of the cone to the point $X$ in $C(X)$ and maps the base of the cone onto the set of singletons in $C(X)$.

1. Property $K$ and the cone $=$ hyperspace property. A continuum $X$ is said to have property $\mathrm{K}$ at a point $a \in X$ provided that, given any $\varepsilon>0$, there exists a $\delta>0$ such that if $b \in X$ with $d(a, b)<\delta$ and $a \in A \in C(X)$, then there exists $B \in C(X)$ such that $b \in B$, and $\rho(A, B)<\varepsilon$.

A continuum $X$ has property $\mathrm{K}$ if and only if it has property $\mathrm{K}$ at each point.

THeOReM 1. Let $X$ be a continuum with property $K$, and let $Z$ be a proper nondegenerate subcontinuum of $X$. Suppose each arc component of $X$ is the one-to-one continuous image of the real line such that the images of $[0, \infty)$ and $(-\infty, 0]$ are dense in $X$. If $G\left(a, t_{1}\right)=Z=G\left(b, t_{2}\right)$, then $a=b$.

Proof. Each proper, nondegenerate subcontinuum of $X$ is an arc, so $Z$ is an arc in $X$ containing $a$ and $b$. Assume $a \neq b$ and $t_{1} \geqslant t_{2}$. Order the arc component containing $Z$ such that $a<b$. There exists a point $c<a$ such that $\mu([c, a])=t_{1}$. Then no $\operatorname{arc} B$ with $\mu(B)=t_{2} \leqslant t_{1}$ contains both $b$ and $c$. This contradiction implies $a=b$.

COROLlaRY 2. Let the continuum $X$ satisfy the hypotheses of Theorem 1 and have the covering property. For each $t<1$, the map $H_{t}: X \rightarrow \mu^{-1}(t)$ is a homeomorphism. In particular, $X$ is Whitney stable.

Proof. By Theorem 1 , the map $H_{t}$ is one-to-one. Since $X$ has the covering property, $H_{t}$ is surjective [3].

COROllary 3. Let $X$ satisfy the hypotheses of Corollary 2. Then the map $\hat{H}$ : $\operatorname{Cone}(X) \rightarrow C(X)$ is a homeomorphism; hence $X$ has the cone = hyperspace property.

Corollary $4[5,9]$. Solenoids have the cone = hyperspace property.

Proof. Solenoids belong to Class (W) [5], and solenoids have property $\mathrm{K}$.

THEOREM 5. Suppose each arc component of the continuum $X$ is the one-to-one continuous image of the real line such that the images of $[0, \infty)$ and $(-\infty, 0]$ are dense in $X$. Suppose each arc in $X$ lies in an open set homeomorphic with Cartesian product of Cantor set and an open interval. Then $X$ has property $K$.

Proof. We show that $X$ has property $\mathrm{K}$ at the point $p$ in $X$. Let $\varepsilon>0$. Let $A=[a, b]$ be an arc containing $p$ such that each of the subarcs $[a, p]$ and $[p, b]$ are of Hausdorff distance less than $\varepsilon$ from $X$. Let 0 be an open set containing $A$ and 
homeomorphic with the product of a Cantor set and an open interval. There exists $\delta>0$ such that $B(p ; \delta) \subseteq 0$ and such that if $d(p, q)<\delta$ and $B$ is a subcontinuum of 0 containing $p$, then there exists a continuum $C$ in 0 such that $q \in C$ and $\rho(B, C)<\varepsilon$. This is the desired $\delta$, for if the subcontinuum $B$ of $X$ contains $p$ but is not a subset of 0 , then choose $C=X$ because $\rho(B, X)<\varepsilon$.

THEOREM 6. There exists a continuum with the cone = hyperspace property that is neither arc-like nor circle-like.

Proof. The plane continuum described by Bing [1, p. 222] has four complementary domains. Hence, it is neither arc-like nor circle-like. It satisfies the hypotheses of Theorem 5, so it has property K. Furthermore, each arc component of $X$ is the one-to-one continuous image of the real line and both ends are dense in $X$. Hence, $X$ satisfies the hypotheses of Theorem 1. Thus, the map $\hat{H}: \operatorname{Cone}(X) \rightarrow C(X)$ is a homeomorphism provided we show that $X$ has the covering property.

To show that $X$ has the covering property, we verify the three hypotheses of [5, Corollary 4.7]. Each proper, nondegenerate subcontinuum of $X$ is an arc. No subcontinuum of $X$ locally separates two points. Finally, $X$ has finite open covers of arbitrarily small mesh whose nerves are graphs with Betti number four. Hence, $X$ has the covering property, and the proof of the theorem is complete.

Question. Suppose the finite-dimensional continuum $X$ has the cone $=$ hyperspace property. Must $X$ have property K? Must $X$ be Whitney stable? Must $X$ have the covering property?

Had the property that every proper nondegenerate subcontinuum is an arc been a Whitney property, we would not have had to require that $X$ have the covering property in Corollaries 2 and 3. We investigate this property next.

THEOREM 7. The property of having every nondegenerate proper subcontinuum be an arc is a Whitney property for the class of continua with the covering property.

Proof. Let $X$ be a continuum with the covering property. Let $Z$ be a proper, nondegenerate subcontinuum of $\mu^{-1}(t)$. Since $X$ has the covering property, $\sigma(X)$ is a proper, nondegenerate subcontinuum of $X$, and hence an arc. So $Z$ is a subcontinuum of $\mu^{-1}(t) \cap C(\sigma(Z))$. Hence, $Z$ is an arc, because Whitney levels of arcs are arcs.

Finally, we give an example to show that the restriction to continua with the covering property is necessary in the above theorem.

EXAMPLE 1. Let $X$ be the indecomposable chainable continuum pictured in [7, p. 205] and obtained as the inverse limit of arcs with each bonding map being the open map of $I$ onto $I$ pictured in Diagram II.

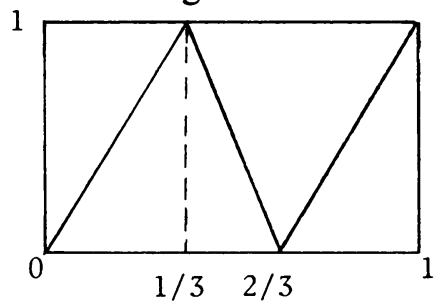

Diagram II 
Every proper nondegenerate subcontinuum of $X$ is an arc, and $X$ has exactly two endpoints. Let $Y$ be the circle-like continuum obtained by identifying these two endpoints. Each proper nondegenerate subcontinuum of $Y$ is an arc. For each $t$ between 0 and 1, however, $\mu^{-1}(t)$ is the union of an $\operatorname{arc} A$ and an indecomposable continuum $B$ (actually $B$ is homeomorphic to $X$, by the techniques of this paper) such that $A \cap B$ is the common set of endpoints of $A$ and $B$. Hence, $Y$ has the property that for any Whitney map $\mu$ and any $t, 0<t<1, \mu^{-1}(t)$ contains a proper nondegenerate subcontinuum that is not an arc.

2. Continua with arcs as subcontinua. Let $X$ be a continuum such that each proper, nondegenerate subcontinuum is an arc. A point $e$ of $X$ is called an endpoint of $X$ if $e$ is an endpoint of every arc that contains it.

In the next theorem, we show that, for certain continua, $H_{t}$ is a monotone map; it follows from the proof that the number of points with nondegenerate inverses is no more than the number of endpoints of $X$.

THEOREM 8. Suppose the continuum $X$ has property $K$. If each proper, nondegenerate subcontinuum of $X$ is an arc, then $H_{t}$ is a monotone map, for each $t$.

Proof. Suppose $a$ and $b$ are points of $X$ with $H_{t}(a)=H_{t}(b)$. Let $Z \in C(X)$. The cases $Z=X$ or $Z$ is a singleton (i.e., $t=1$ or $t=0$ ) are trivial, for $H_{1}$ is a constant map and $H_{0}$ is a homeomorphism. Let us assume, therefore, that $Z$ is a proper, nondegenerate subcontinuum of $X$. Then $Z$ is an $\operatorname{arc}$ in $X$ containing $a$ and $b$.

The cases $X=S^{1}$ and $X=I$ are simple, and the proofs will be omitted. If $X$ is neither a circle nor an arc, then $X$ is an indecomposable continuum such that each arc component of $X$ is dense in $X$, since the composants of $X$ are the arc components.

There exist numbers $t_{1}$ and $t_{2}$ such that $G\left(a, t_{1}\right)=G\left(b, t_{2}\right)$. Assume $t_{2} \leqslant t_{1}$. Let $[a, b]$ be the subarc of $Z$ irreducible from $a$ to $b$. Let $d$ be a point such that $a \leqslant d \leqslant b$. We shall prove the existence of a number $t_{3}$ such that $G\left(d, t_{3}\right)=Z$; hence, $H_{t}(d)=Z$ and $H_{t}$ is monotone.

If there exists a point $c$ such that $\mu([c, a])=t_{1}$, then the proof of Theorem 1 shows that $a=b$. Otherwise, there exists an endpoint $e$ of $X$ such that $\mu([e, a])<$ $t_{1}$. Since the arc component containing $a$ is dense in $X$, there exists a point $z$ such that $\mu([a, z])=t_{1}$. So $Z=[e, z]$. It follows that $\mu([b, z])=t_{2}$. Let $t_{3}=\mu([d, z])$. Then $t_{2} \leqslant t_{3} \leqslant t_{1}$. Also $\mu([e, d]) \leqslant t_{3}$. Hence, $G\left(d, t_{3}\right)=Z$.

COROllary 9. Let the continuum $X$ satisfy the hypotheses of Theorem 7. Then, the map $\hat{H}: \operatorname{Cone}(X) \rightarrow C(X)$ and, for $t<1$, the map $H_{t}: X \rightarrow \mu^{-1}(t)$ are cell-like maps (in fact, each nondegenerate point inverse is an arc). Furthermore, if $X$ belongs to Class $(W)$, then both maps are surjective.

The following example shows that $H_{t}$ is not always monotone.

Let $X$ be the space pictured in Figure 1. Analytically, $X=A \cup B \cup C$, where

$$
\begin{aligned}
& A=\left\{(x, y):(x+2)^{2}+y^{2}=1\right\}, \\
& B=\left\{(x, y): x^{2}+y^{2}=1\right\}, \text { and } \\
& C=\left\{(x, y):(x-2)^{2}+y^{2}=1\right\} .
\end{aligned}
$$


Let $\mu: C(X) \rightarrow[0,6 \pi]$ be defined by arc length, and $Z=\{(x, y) \in X:-2<x<$ 2 ). The subcontinuum $Z$ is pictured in Figure 1.

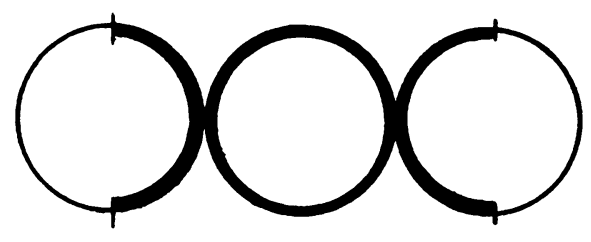

Figure 1

Then $G((0,1), \pi)=Z=G((0,-1), \pi)$, but $G((x, y), t) \neq Z$ for any $(x, y) \in$ $X \backslash\{(0,1),(0,-1)\}, t \in[0,6 \pi]$. Hence $H_{4 \pi}^{-1}(Z)=\{(0,1),(0,-1)\}$ which is not connected. Therefore $H_{4 \pi}$ is not monotone.

Question. Is $H_{t}$ monotone for each $t$ whenever $X$ is hereditarily unicoherent and atriodic?

Next we apply Theorem 8 to investigate the structure of the hyperspace of certain chainable continua. John Walsh has pointed out to us that decomposition theorists have considered decompositions such as that of Theorem 11 and called them sliced decompositions (cf. [2]).

The chainable continua of interest are those obtainable as the inverse limits of arcs with open bonding maps. The chainable continuum used in Example 1 is such a continuum. In [7, p. 205] are pictured two such continua, one with one endpoint and one with two endpoints. Such continua must have either one or two endpoints. Every proper nondegenerate subcontinuum of these continua is an arc. Furthermore, these continua have property K. Will Watkins and Wojciek Debski independently have classified such continua and have shown that there are $c=2^{\omega_{0}}$ topologically distinct ones.

THEOREM 10. Suppose $X$ is the inverse limit of arcs with open bonding maps. Then $X$ is Whitney stable.

Proof. For each $t$ between 0 and 1, the map $H_{t}: X \times\{t\} \rightarrow \mu^{-1}(t)$ is a surjective cell-like map with exactly as many nondegenerate point inverses as $X$ has endpoints (namely, one or two). Furthermore, each of these nondegenerate point inverses is an arc, and each such arc $A$ is contained in a closed set $W$ homeomorphic to the product of $I$ and the Cantor set. Such a set $W$ may be chosen with the additional properties that: (1) exactly one endpoint of $A$ is an endpoint of a maximal arc in $W$, (2) the boundary of $W$ in $X$ is the set of endpoints of maximal arcs in $W$, and (3) if $X$ has two endpoints, then the two chosen sets $W_{1}$ and $W_{2}$ are disjoint.

The point inverses of $H_{t}$ yield an (very simple) upper semicontinuous cell-like decomposition of $X \times\{t\}$. The facts in the previous paragraph imply that the decomposition is shrinkable; this means that, for each $\varepsilon>0$, there exists a homeomorphism $h$ of $X \times\{t\}$ onto itself such that (a) $\rho\left(H_{t}(Z), H_{t}(h(Z))\right)<t$ for each $Z$ in $X \times\{t\}$ and (b) diameter $\left(h\left(A_{i}\right)\right)<\varepsilon$, for each nondegenerate point inverse $A_{i}$. In fact $h$ can be chosen to be the identity map on the complement of $W_{1} \times\{t\}$ (and $W_{2} \times\{t\}$ if $W_{2}$ exists). 
The Bing Shrinkability Criterion [2] implies that $H_{t}$ can be approximated by homeomorphisms. Hence, $\mu^{-1}(t)$ is homeomorphic to $X$.

THEOREM 11. Suppose $X$ is the inverse limit of arcs with open bonding maps. Then there is a homeomorphism $L: \operatorname{Cone}(X) \rightarrow C(X)$ making the following diagram commute:

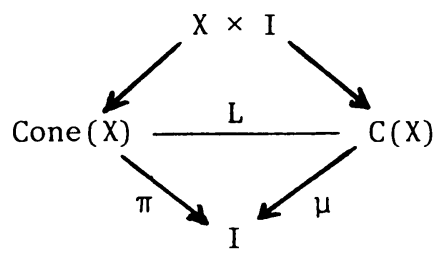

\section{Diagram III}

Proof. The idea of this proof is that the approximations of $H_{t}$ by Homeomorphisms in the previous theorem may be chosen to form the desired homeomorphism $L$. It is worth noting that no such homeomorphism $L$ exists for a pseudo-arc, even though the pseudo-arc is Whitney stable and $H_{t}$ is a cell-like map. The shrinkability of the decomposition is essential.

The proof is similar to that of the previous theorem. We wish to approximate the restriction of $H$ to $X \times[1-1 / n, 1-1 /(n+1)], n$ a natural number, by homeomorphisms that agree on the intersection of two such sets. As in the previous theorem, the homeomorphism will be the identity map on the complement of certain sets analogous to the sets $W$. The sets $W$ of the previous theorem are now replaced, however, by sets homeomorphic to the product of a two-cell and the Cantor set. Each nondegenerate element of the decomposition lies in some set of the form $X \times\{t\}$; hence, in the terminology of [2], the decomposition is sliced. In a two-cell that contains nondegenerate elements of the decomposition, the decomposition is similar to shrinking to points all horizontal line segments to the left of the diagonal line segment in the two-cell in Diagram IV.

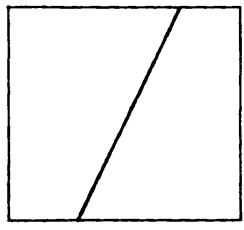

Diagram IV

The approximation by homeomorphisms may be done in such a way that the lower triangle of Diagram III commutes. This completes the proof.

COROLlARY 12. If $X$ is the inverse limit of arcs with open bonding maps, then $X$ has the cone = hyperspace property.

THEOREM 13. Let $X=A \cup B$ where

$$
A=\{(x, y): x=0,-1<y<1\}, \quad B=\{(x, y): x>0, y=\sin 1 / x\},
$$


and let $Y=C \cup D$ where

$$
C=\left\{(x, y): x^{2}+y^{2}=1\right\}, \quad D=\left\{[1+1 / t] e^{i t}: t \geqslant 1\right\} .
$$

Then neither $X$ nor $Y$ admits essentially different Whitney maps.

Proof. The proof of Theorem 8 shows that for $t<\mu(A)$ (respectively $\mu(C)$ ), the only nondegenerate point inverses under $H_{t}$ are those which contain an endpoint. Furthermore, each endpoint of $X$ (respectively, the endpoint in $Y$ ) is contained in an arc, which is the nondegenerate point inverse containing that endpoint. Hence, point inverses of $H_{t}$ are arcs or points. Also, $\mu^{-1}(t)$ is irreducible, so it is the image of $X$ (respectively, $Y$ ) under the map $H_{t}$. Such monotone images are clearly homeomorphic to $X$ (respectively $Y$ ). (See also [8].)

If $t \geqslant \mu(A)$ (respectively $\mu(C)$ ) and $t<1$, then $H_{t}$ has exactly two nondegenerate point inverses, one containing $A$ (respectively $C$ ) and one containing an endpoint. The image of such a monotone map is an arc. Since $H_{t}$ is surjective, $\mu^{-1}(t)$ is an arc.

Finally, if $t=1$, then $H_{t}$ is a constant map with a point as its image.

\section{REFERENCES}

1. R. H. Bing, A simple closed curve is the only homogeneous bounded plane continum that contains an arc, Canad. J. Math. 12 (1960), 209-230.

2. Robert J. Daverman and D. Kriss Preston, Shrinking certain sliced decompositions of $E^{n}+1$, Proc. Amer. Math. Soc. 79 (1980), 477-483.

3. A. Dilks, Contractible hyperspaces are arc-smooth at the top (to appear).

4. __ Structure of hyperspaces, Dissertation, Tulane University, New Orleans, 1980.

5. J. Grispolakis and E. D. Tymchatyn, Continua which are continuous images of weakly confluent mappings only, Houston J. Math. 5 (1979), 483-502.

6. J. L. Kelley, Hyperspaces of a continum, Trans. Amer. Math. Soc. 52 (1942), 22-26.

7. K. Kuratowski, Topology, vol. II, Academic Press, New York, 1968.

8. S. B. Nadler, Jr., Confluent images of the sinusoidal curve, Houston J. Math. 3 (1977), 515-519.

9. ___ Hyperspaces of sets, Dekker, New York, 1978.

Mathematical Sciences, McNeese State University, Lake Charles, Louisiana 70609

Department of Mathematics, Tulane University, New Orleans, Louisiana 70118 